\title{
Alpha and beta oscillatory activity during a sequence of two movements
}

\author{
Manuel Alegre ${ }^{\mathrm{a}}$, Iñaki García de Gurtubay ${ }^{\mathrm{a}}$, Alberto Labarga ${ }^{\mathrm{b}}$, \\ Jorge Iriarte ${ }^{\mathrm{a}}$, Armando Malanda ${ }^{\mathrm{c}}$, Julio Artieda ${ }^{\mathrm{a}, *}$ \\ ${ }^{a}$ Clinical Neurophysiolgy Section, Department of Neurology, Clínica Universitaria, Facultad de Medicina, \\ Universidad de Navarra, Avda, Pío XII, 36, 31008 Pamplona, Spain \\ ${ }^{\mathrm{b}}$ Department of Automation and Computer Science, Escuela Superior de Ingeniería de Telecomunicación, \\ Universidad Pública de Navarra, Campus de Arrosadia, 31006, Pamplona, Spain \\ ${ }^{\mathrm{c}}$ Department of Electrical and Electronic Engineering, Escuela Superior de Ingeniería de Telecomunicación, \\ Universidad Pública de Navarra, Campus de Arrosadia, 31006, Pamplona, Spain
}

Accepted 5 September 2003

\begin{abstract}
Objective: We studied movement-related electroencephalographic oscillatory changes in the alpha and beta range during a sequence of two movements in 7 healthy volunteers, in order to investigate the relationship between these changes and each component in the sequence.

Methods: The sequence consisted of a wrist active extension-passive flexion followed by a first and second finger pincer. A total of $10.5 \mathrm{~s}$ sweeps were recorded using the level of surface electromyographic (EMG) activity in wrist extensors as trigger, including a $7.5 \mathrm{~s}$ prestimulus. The sweeps were also realigned manually offline using as trigger the end of the first EMG burst, or the beginning of the second movement. An index of the changes in non-phase-locked energy in the 7-37 Hz range was obtained by averaging single-sweep timefrequency transforms.
\end{abstract}

Results: The duration of each of the movements in the sequence and the relationship between them were compatible with the use of two different motor programmes in the sequence. In the beta band, a decrease in energy (event-related desynchronisation, ERD) began $1.5 \mathrm{~s}$ before the onset of the first movement, and was sustained until the end of the second movement. No energy increases were observed until the end of the second movement. In the alpha band, the ERD began 0.5 seconds before the first movement and was sustained throughout the recording.

Conclusion: These findings suggest that the beta-event-related synchronisation is related to the end of the whole motor process, and not to the end of each motor programme.

(C) 2004 International Federation of Clinical Neurophysiology. Published by Elsevier Ireland Ltd. All rights reserved.

Keywords: Event-related desynchronisation; Event-related synchronisation; Sequential movements; Human; Electroencephalogram

\section{Introduction}

Cortical oscillatory activity has gained relevance during the last decade, as it might represent a general mechanism in perceptual and motor binding (Singer, 1993; Farmer, 1998). A voluntary movement is accompanied by changes in cortical activity that can be detected in the electroencephalogram (EEG) (Chatrian et al., 1959). The disappearance of the central mu rhythm (in the alpha range) during movement is clearly visible in some subjects without additional processing (Gastaut et al., 1954; Chatrian et al., 1959). But these changes are not limited to the alpha band. Using mainly Fourier-based mathematical approaches,

\footnotetext{
* Corresponding author. Tel.: + 34-948-255-400; fax: + 34-948-296-500

E-mail address: jartieda@unav.es (J. Artieda).
}

the changes in alpha and beta activity during different self-initiated movements have been studied in detail and quantified by several groups. In the alpha band, a decrease in energy begins about $1 \mathrm{~s}$ before the movement (alpha eventrelated desynchronisation, ERD), is sustained until the end of it, and is followed by a slow increase after movement (alpha event-related synchronisation, ERS) (Pfurtscheller and Aranibar, 1979; Derambure et al., 1993). In the beta band, a fall in activity which begins more than $1 \mathrm{~s}$ before movement and lasts till the end of muscle contraction (betaERD), and a post-movement rebound over baseline level (beta-ERS), have been thoroughly described (Pfurtscheller, 1981; Pfurtscheller et al., 1996; Derambure et al., 1999; Alegre et al., 2003). The exact significance of these changes has not been clearly established, though. Pre-movement 
changes (mainly in the beta band), have been related to motor preparation, while the post-movement ERS has been related either to sensory afferences (Cassim et al., 2001), the return of the motor cortex to a 'rest' state (Pfurtscheller et al., 1996), or to phenomena related to the closure of the motor process (Alegre et al., 2002).

The aim of our work was to analyse the cortical energy changes in the alpha and beta band during a sequence of two movements, studying the relationship of these changes with the beginning and the end of each of the movements. A complex sequential movement can be due to a single motor program or to a combination of different motor programmes; in the latter case the relationship between the oscillatory changes and each motor program could also be established. If the energy changes are related to each motor program, a summation of the changes corresponding to each program could be expected. Alternatively, these changes could be related to a higher order movement plan, including the whole sequence. In this case, the changes observed would correspond to a single ERD/ERS pattern.

\section{Methods}

\subsection{Signal recording}

A total of 7 healthy right-handed volunteers ( 5 men, two women) with an age range between 25 and 37 years were studied. All of them gave their written consent after a detailed explanation of the procedure, previously approved by the institutional ethics committee.

The subjects were comfortably sat in a dimly-lit soundattenuated room with eyes opened looking at a fixed point. Nineteen surface $\mathrm{Ag} / \mathrm{AgCl}$ recording electrodes were placed in positions within the 10-20 system (Jasper, 1958). All impedances were systematically kept below $5 \mathrm{~K} \Omega$. Both linked earlobes were used as reference in the recording; a reference-free montage (intrinsic Hjorth laplacian) was computed afterwards. EEG channels were amplified $\times 20,000$, with low-pass and high pass filters set at 100 and 0.1 or $0.3 \mathrm{~Hz}$, respectively. All channels were digitised at $200 \mathrm{~Hz}$ and stored in a PC for offline analysis.

The subjects were instructed to perform self-paced sequences of two movements, wrist extension and digital pincer, with a random interval between sequences longer than $11 \mathrm{~s}$. Specifically, the subjects were told to begin the second movement only after the first one had ended completely.

\subsection{Signal processing}

A minimum of 100 sweeps per subject were recorded, between $7.56 \mathrm{~s}$ before and $2.56 \mathrm{~s}$ after the beginning of the first movement, using a level trigger in an extensor carpi surface EMG channel. All samples with visible artefacts were rejected manually. An off-line pre-processing was performed, obtaining two asymmetrical sections from each sweep. The first sections (named 'rest', $2.56 \mathrm{~s}$ of duration) were taken from the beginning of the sweeps after discarding the initial $100 \mathrm{~ms}$. The second group of sections (named 'movement') included the $5.12 \mathrm{~s}$ period between $2.56 \mathrm{~s}$ before and $2.56 \mathrm{~s}$ after the trigger.

Two different realignments were performed offline. First, all sweeps were manually realigned using the end of the first movement as trigger, instead of the beginning. Also, all sweeps were also realigned using the beginning of the second movement as trigger. All the procedures described (pre-processing in two sections, time-frequency analysis and statistics) were applied to the 3 groups of sweeps (triggered by the beginning of the first movement, triggered by the end of the first movement, triggered by the beginning of the second movement).

An offline realignment was also performed on a series of single ballistic movements (brisk wrist extension, as in the first movement of the sequence) performed by a group of 10 volunteers, described in a previous study (Alegre et al., 2003). These sweeps were realigned manually using the end of the rectified surface EMG discharge as trigger; the same analysis procedure described below was applied to them.

\subsection{Time-frequency and statistical analysis}

Oscillatory activity associated with perceptual or motor phenomena may appear with a certain jitter in latency from trial to trial. When all trials are averaged (as in the classic evoked potentials), all activity not coincident in phase tends to disappear. Therefore, the time-frequency representation of the average of all trials only represents oscillatory activity clearly phase-locked to the trigger of the sweep. However, if the time-frequency transform is computed for every single trial and averaged afterwards, all oscillatory activity is added despite its phase, as the values of energy are always positive. Processing each response separately, without averaging, implies that the interesting elicited components are several orders of magnitude smaller than other rhythmical activities in the EEG, so efficient mathematical tools are required for a good visualisation.

Our method combined a multiresolution wavelet algorithm for signal extraction and the Gabor transform to represent the temporal evolution of the selected frequency components (Cohen, 1989). Wavelet-based digital filters were used to extract the desired frequency components $(7-37 \mathrm{~Hz})$, and a discrete Gabor expansion was used to represent the time-frequency distribution of the energy in the reconstructed signal in a 2-D coloured graph. A more detailed description of the procedure can be found elsewhere (Gurtubay et al., 2001).

The average of the transforms for the movement was divided by the mean energy for each frequency during rest. In order to avoid discontinuity effects, only the 4 central seconds were displayed. The energy changes in the most 
significant frequency bands were also linearly displayed for an easier visualisation.

All individual sweeps from the rest and movement groups were compared using a Wilcoxon test (energy values do not have a gaussian distribution (Tallon-Baudry et al., 1996)), and the statistical significance of the comparison for small windows of time-frequency domain represented in a logarithmic scale using a dual colour scheme (red for decreases, blue for increases). Significance values of the same time-frequency range in different electrodes were also represented over a 2-D model of the head.

This mathematical procedure (TF transform averaging and normalisation, and statistical comparison) was applied to each individual subject and to each group as a whole.

\section{Results}

\subsection{Movement timing and slow potentials}

The mean duration of the first movement was $571 \mathrm{~ms}$, with individual means ranging from 378 to $713 \mathrm{~ms}$. The mean duration of the second movement was $661 \mathrm{~ms}$ with individual means between 409 and 1067 ms. No correlation was found between the length of the first and the second EMG bursts in the sequence in any of the subjects $(-0.15<r<0.15, P=\mathrm{ns})$ in all cases in the Spearman test. This finding suggests that the sequential movement was due to two different motor programmes (Benecke et al., 1986). The interval between the onset of both movements was higher than $200 \mathrm{~ms}$ in all subjects, and therefore also compatible with the execution of two different programmes. The mean interval between the end of the first movement and the beginning of the second was $242 \mathrm{~ms}$, with an individual mean range between 75 and $498 \mathrm{~ms}$.

Although limited by the filter settings, a pre-movement potential was observed in all cases before the first movement, with the typical components (readiness potential, negative slope) and topography.

\subsection{Beta energy changes}

A decrease in beta energy (beta-ERD, range 17-30 Hz) was observed between 1.5 and $2 \mathrm{~s}$ before the beginning of the first movement in the central region $(85 \%$ of baseline at $23 \mathrm{~Hz})$. This decrease was initially limited to $\mathrm{C} 3$, but it became significant in both sides (C3 and C4) $0.5 \mathrm{~s}$ before the movement (Fig. 1). Near the beginning of it, the ERD decreased notably and became symmetrically bilateral, extending to frontal and parietal regions. The ERD was sustained in similar values until the end of the second movement, when it was followed by a ERS, maximum in C3 $(170 \%$ at $18-20 \mathrm{~Hz})$. The peak of the ERD was at a frequency higher than that of the ERS. Minimum ERD values were observed in the central region, bilaterally (63\% at $21 \mathrm{~Hz}$ in $\mathrm{C} 3$ and $\mathrm{C} 4)$.

The pattern observed was similar after realigning the sweeps using the beginning of the second movement, or the end of the first movement as trigger (see Figs. 2 and 3). Fig. 2 compares the results obtained using the end of the (first) movement as trigger in a single movement and in the sequence of two movements. After a single ballistic movement, the beta energy increased progressively after the trigger reaching a maximum value $(175 \%) 1.1 \mathrm{~s}$ after it. The slope of the increase (ERS) after the single ballistic movement was higher than the slope of the movementrelated ERD. In the sequence of two movements, no ERS

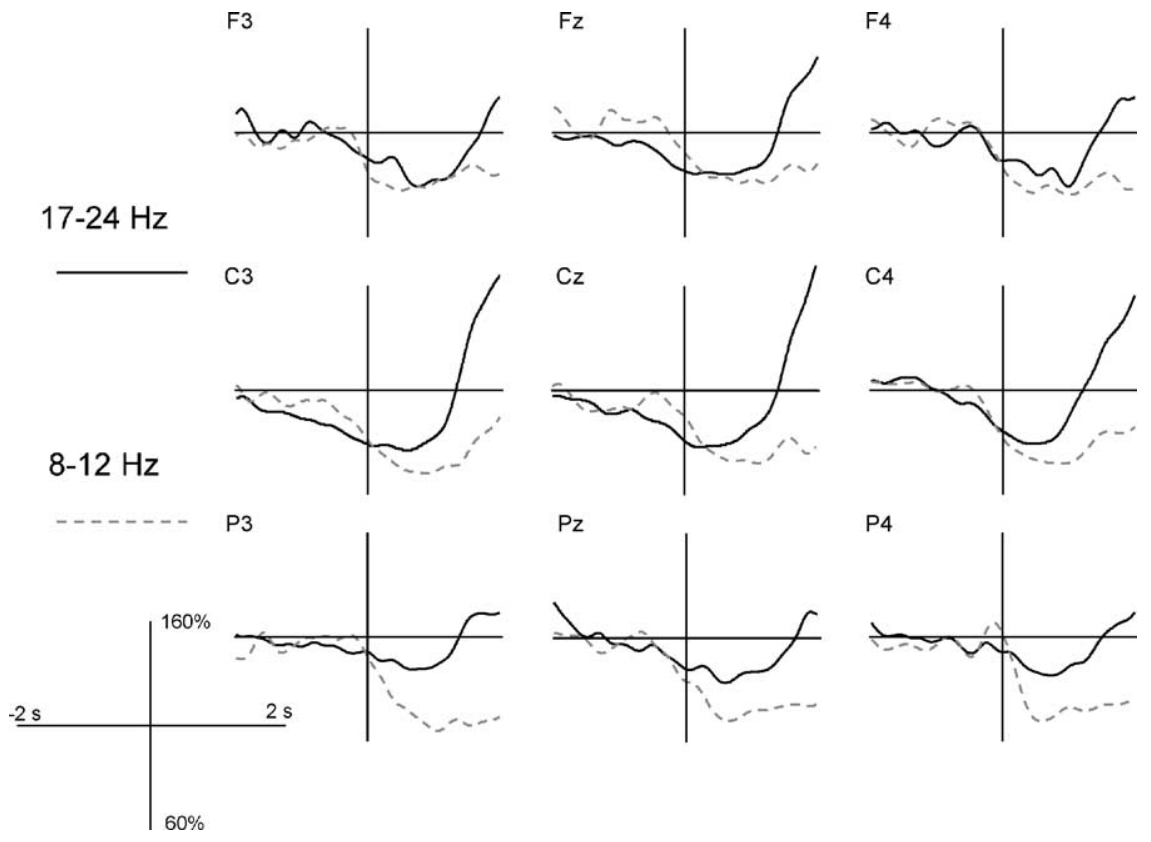

Fig. 1. Linear representation of the changes observed during the performance of the sequential movement, in the two most reactive bands (alpha and beta), in 9 different electrodes. The vertical line represents the onset of the first movement (used as trigger), and the horizontal line represents baseline energy values. 
A

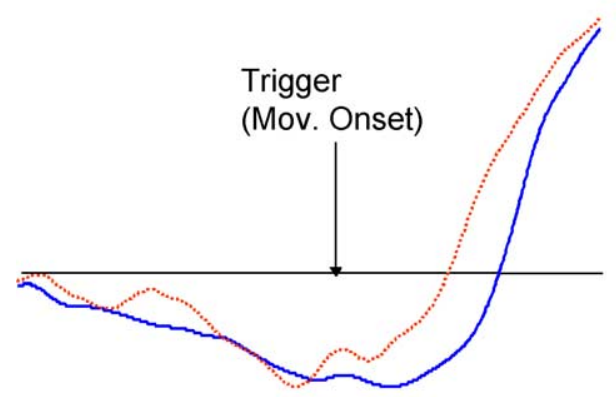

B

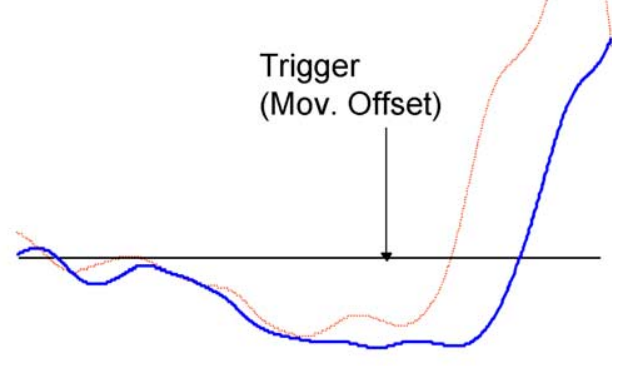

$-2$

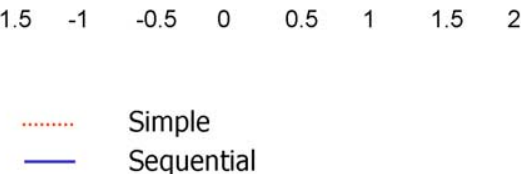

Fig. 2. Comparison of the beta changes observed in C3 during the sequential movement with those observed during a simple ballistic one. The traces on the left were obtained using the beginning of the first movement as trigger (time $=0$, vertical arrow). The traces on the right were manually triggered offline by the end of the first movement (time $=0.5$, vertical arrow)
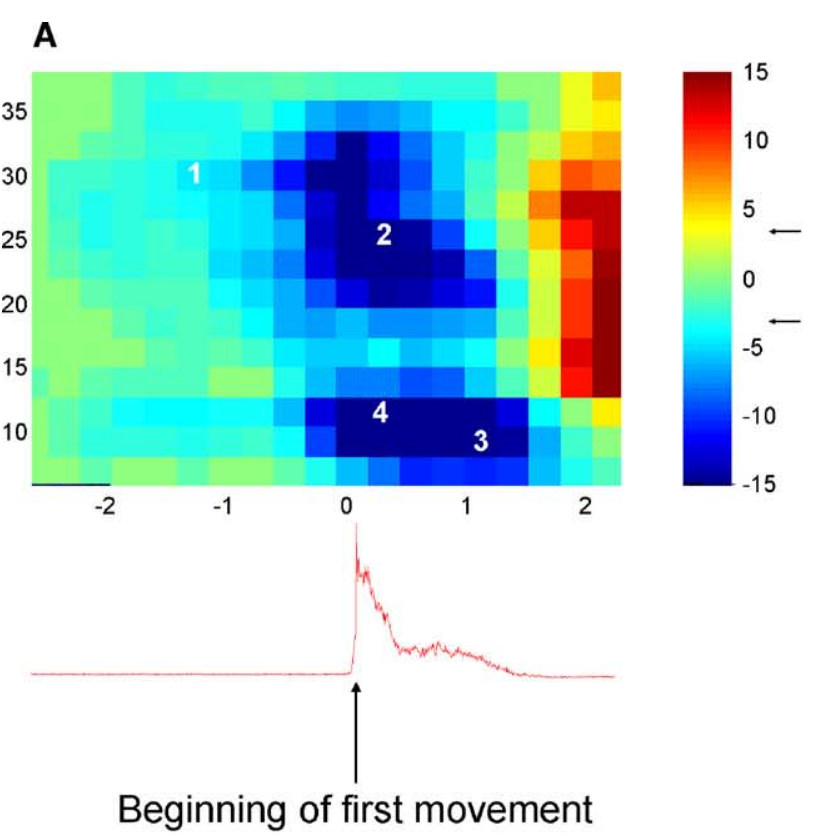

C

1

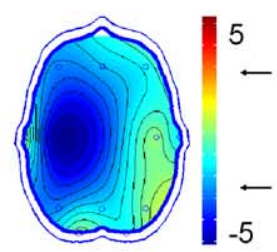

2

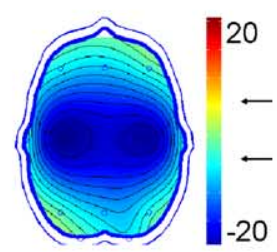

\section{B}
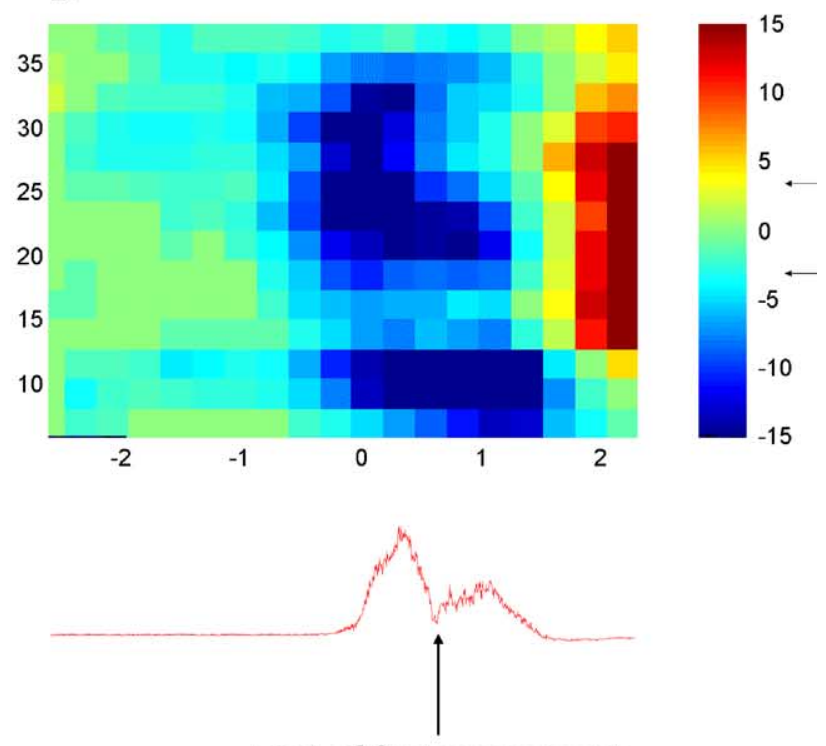

End of first movement
3

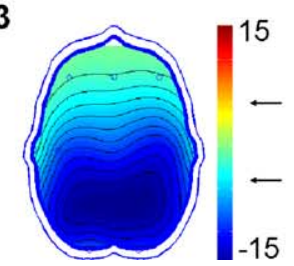

4

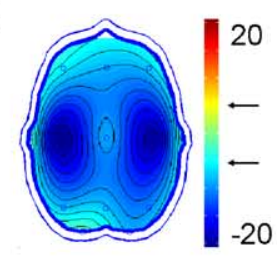

Fig. 3. Statistical significance of the changes observed during the performance of the sequential movement. The colour scale represents the uncorrected statistical significance (Wilcoxon test) in a logarithmic scale $( \pm \log P$ ). Negative values (blue) correspond to significant decreases, and positive values (red) correspond to significant decreases. Horizontal arrows indicate a corrected value of $P=0.05$ (Bonferroni correction: corrected $P=[$ uncorrected $P *$ number of comparisons]). The top left figure (A) represents the changes in alpha and beta activity in C3 (contralateral central electrode) with the sweeps aligned using the beginning of the first movement as trigger (time 0, vertical arrow). The top right figure (B) represents the changes observed in the same electrode when the sweeps are aligned using the end of the first movement as trigger (time $0.5 \mathrm{~s}$, vertical arrow). The lower part of the figure (C) represents the topography of the statistical significance of the changes at different times and frequencies. The numbers indicate the time-frequency points in the upper left graph. 
was observed after the end of the first one; on the opposite, the ERD was maintained until the end of the second movement, indicating that the first movement in the sequence did not generate any ERS. The statistical significance of the beta ERD was slightly lower, but with a similar temporal pattern. When the sweeps were aligned with the beginning of the second movement, only minimal changes in the temporal evolution of the ERD were observed.

\subsection{Alpha energy changes}

A decrease in energy in the alpha band (ERD) was observed between 1 and $0.5 \mathrm{~s}$ before the onset of the first movement. The topography of this decrease was different for low (8-10) and high (10-12) alpha frequencies. In the lower range, the changes were predominantly posterior (parietal and occipital), and more diffuse. In the higher range, the changes were maximum in centro-parietal regions, limited to lateral electrodes, and started earlier. In all electrodes, the decrease was more marked than in the beta range. The highest significance values and the minimal energy values were around $9 \mathrm{~Hz}$ (55\% of baseline). The decrease was sustained thorough the recording, although a tendency to recovery was observed, mainly in frontal leads.

As in the beta band, the changes observed after realigning the sweeps using the end of the first movement or the beginning of the second movement as trigger were minimal; in the latter case the minimum value of the ERD was displaced towards the second movement.

\section{Discussion}

A beta-ERS has been thoroughly described after simple ballistic movements (Pfurtscheller et al., 1998). ERS values are higher when the sweeps are aligned with the end of the movement (Pfurtscheller et al., 1996). A beta ERD/ERS pattern has also been described during and after negative movements (induced by muscle relaxation) in magnetoencephalographic (MEG) and EEG recordings (Toma et al., 2000; Alegre et al., 2003). Passive movements are also followed by an ERS (Cassim et al., 2001; Alegre et al., 2002). Moreover, a beta-ERS is present after either somatosensory or mechanical stimulation of the limbs, without any movement (Salenius et al., 1997; Pfurtscheller et al., 2001). Some authors have proposed, based on these findings, that the post-movement beta-ERS might be related to the sensory afferences originated by the movement itself. Alternatively, the post-movement beta-ERS might be due to processes related to the closure of the motor act. The main finding in our study is that the post-movement beta-ERS does not appear after the first movement in a sequence of two motor programmes. Instead of that, the beta ERD that appears before movement onset is sustained until the end of the second movement in the sequence. Previous studies on
ERD in sequential movements had been just limited to the global sequence, and not to each component (Manganotti et al., 1998; Deiber et al., 2001).

Sequential movements can be due to a single motor program, or to a sequence of motor programmes. Typical examples of single-program motor sequences are signing, typing (Terzuolo and Viviani, 1980) or pronouncing a word (Tuller et al., 1982). A sequential movement due to a single motor programme must have a constant rate of durations between each movement in the sequence (Carter and Shapiro, 1984). We found no correlation between the length of the EMG burst in each of the two movements, suggesting that they were due to two different motor programmes. Also, the latency between the beginning of both movements goes against a single motor programme, as it was higher than $200 \mathrm{~ms}$ in all subjects (Benecke et al., 1986).

A sequential movement consisting of two different motor programmes could be considered just the result of the assembly of the two programs. Alternatively, the existence of a motor image or motor plan that includes the whole movement and is in a higher hierarchy than the motor programs has been proposed (Rothwell, 1994). A parallel processing in the brain for serial actions has been suggested in a recent study of neuronal activity in the prefrontal cortex of primates (Averbeck et al., 2002). Our results support these latter hypothesis. The movement was built with two different motor programmes, and the beta ERS only appeared after the second one, indicating that the beta-ERS is related to some cortical process beyond the motor program (as the closure of the motor plan). This could also explain the findings of an absent beta-ERS in sustained movements (Cassim et al., 2000; Alegre et al., 2003). It is unlikely that our results are due to the overlap of a post-movement ERS from the first movement and a pre-movement ERD from the second, as the slope and the amplitude of the ERS, especially when the sweeps are triggered using the end of the movement, are higher than the pre-movement ERD. Also, although the interval between the end of the first movement and the beginning of the second was variable between subjects, the results were homogeneous (same pattern in all subjects irrespectively of the interval).

The subjects were told specifically to wait until the end of the first movement before executing the second one. As no ERS was observed after the end of the first movement, our results suggest that sensory afferences might not be the main cause of the beta post-movementERS. However, although the two EMG bursts corresponding to each movement were clearly separated, we can not completely rule out that in some cases the beginning of the second movement overlapped with the passive relaxation of the wrist, thus generating a continuous single movement.

Movement-related potentials (MRPs) have more amplitude before a sequence of movements than before 
a simultaneous movement (Kitamura et al., 1993). This has been related to a higher activation of the supplementary motor area, as shown in positron emission tomography (PET) and function magnetic resonance imaging (fMRI) studies (Shibasaki et al., 1993; Cunnington et al., 1996). The amplitude of the pre-movement ERD was also higher in the sequential paradigm when compared to a single ballistic movement (Fig. 3, left), in accordance with the data from previous studies (Manganotti et al., 1998). This might suggest an origin in the supplementary motor area (at least partially) for the pre-movement beta-ERD, although the topography of the ERD and the MRPs is different.

No alpha-ERS after the first movement was either observed. This could indicate a similar meaning for the alpha and beta-ERS, although the alpha-ERS appears later after the end of the movement and it may have been hidden by the ERD related to the second movement (alpha ERD has more amplitude and slope than alpha ERS). The topography of the alpha changes was quite different for the low alpha band $(8-10)$ and the high alpha band $(10-12 \mathrm{~Hz})$, suggesting that there may be two different alpha rhythms contributing to the alpha-ERD (Pfurtscheller, 1989; Manganotti et al., 1998). The lower alpha ERD has more amplitude in parietal electrodes, and is the responsible for minimum values observed in these areas. The topography of the higher alpha-ERD is more similar to that of the betaERD. The statistical maps (Fig. 1) show that the alpha-ERD begins earlier for the higher frequencies, although these changes were not significant.

In conclusion, our results suggest that alpha and beta energy changes are related to the whole motor act, and not to each component, during a sequence of two movements with two different motor programmes. These findings support the idea of a parallel processing for serial movements.

\section{References}

Alegre M, Labarga A, Gurtubay IG, Iriarte J, Malanda A, Artieda J. Beta EEG changes during passive movements: sensory afferences contribute to the beta event-related desynchronization in humans. Neurosci Lett 2002;331:29-32.

Alegre M, Labarga A, Gurtubay IG, Iriarte J, Malanda A, Artieda J. Movement-related changes in cortical oscillatory activity in ballistic, sustained and negative movements. Exp Brain Res 2003; 148:17-25.

Averbeck BB, Chafee MV, Crowe DA, Georgopoulos AP. Parallel processing of serial movements in prefrontal cortex. Proc Natl Acad Sci USA 2002;99:13172-7.

Benecke R, Rothwell JC, Day BL, Dick JP, Marsden CD. Motor strategies involved in the performance of sequential movements. Exp Brain Res 1986;63:585-95.

Carter MC, Shapiro DC. Control of sequential movements: evidence for generalized motor programs. J Neurophysiol 1984;52:787-96.

Cassim F, Szurhaj W, Sediri H, Devos D, Bourriez J, Poirot I, Derambure P, Defebvre L, Guieu J. Brief and sustained movements: differences in event-related (de)synchronization (ERD/ERS) patterns. Clin Neurophysiol 2000;111:2032-9.
Cassim F, Monaca C, Szurhaj W, Bourriez JL, Defebvre L, Derambure P, Guieu JD. Does post-movement beta synchronization reflect an idling motor cortex? NeuroReport 2001;12:3859-63.

Chatrian GE, Petersen MC, Lazarte JA. The blocking of the rolandic wicket rhythm and some central changes related to movement. Electroenceph clin Neurophysiol 1959;11:497-510.

Cohen L. Time-frequency distributions. A review. Proc IEEE 1989;77: $941-81$

Cunnington R, Iansek R, Bradshaw JL, Phillips JG. Movement-related potentials associated with movement preparation and motor imagery. Exp Brain Res 1996;111:429-36.

Deiber MP, Caldara R, Ibañez V, Hauert CA. Alpha band power changes in unimanual and bimanual sequential movements, and during motor transitions. Clin Neurophysiol 2001;112:1419-35.

Derambure P, Dujardin K, Defebvre L, Bourriez JL, Jacquesson JM, Guieu JD. Etude spatiotemporelle des desynchronisations liees a l'evenement au cours d'une activite motrice autocommandee. Neurophysiol Clin 1993;23:337-51.

Derambure P, Defebvre L, Bourriez JL, Cassim F, Guieu JD. Desynchronisation et synchronisation liees a l'evenement. Etude de la reactivite des rythmes electrocorticaux en relation avec la planification et l'execution du mouvement volontaire. Neurophysiol Clin 1999;29: 53-70.

Farmer SF. Rhythmicity, synchronization and binding in human and primate motor systems. J Physiol Lond 1998;509:3-14.

Gastaut H, Dongier M, Courtois G. On the significance of 'wicket rythms' ('rythmes en arceau') in psychosomatic medicine. Electroenceph clin Neurophysiol 1954;6:687.

Gurtubay IG, Alegre M, Labarga A, Malanda A, Iriarte J, Artieda J. Gamma band activity in an auditory oddball paradigm studied with the Wavelet Transform. Clin Neurophysiol 2001;112:1219-28.

Jasper HH. Report of the committee on methods of clinical examination in electroencephalography. Electroenceph clin Neurophysiol 1958;10: $370-5$.

Kitamura J, Shibasaki H, Takagi A, Nabeshima H, Yamaguchi A. Enhanced negative slope of cortical potentials before sequential as compared with simultaneous extensions of two fingers. Electroenceph clin Neurophysiol 1993;86:176-82.

Manganotti P, Gerloff C, Toro C, Katsuta H, Sadato N, Zhuang P, Leocani L, Hallett M. Task-related coherence and task-related spectral power changes during sequential finger movements. Electroenceph clin Neurophysiol 1998;109:50-62.

Pfurtscheller G. Central beta rhythm during sensorimotor activities in man. Electroenceph clin Neurophysiol 1981;51:253-64.

Pfurtscheller G. Spatiotemporal analysis of alpha frequency components with the ERD technique. Brain Topogr 1989;2:3-8.

Pfurtscheller G, Aranibar A. Evaluation of event-related desynchronization (ERD) preceding and following voluntary self-paced movement. Electroenceph clin Neurophysiol 1979;46:138-46.

Pfurtscheller G, Stancak A, Neuper C. Post-movement beta synchronization. A correlate of an idling motor area? Electroenceph clin Neurophysiol 1996;98:281-93.

Pfurtscheller G, Zalaudek K, Neuper C. Event-related beta synchronization after wrist, finger and thumb movement. Electroenceph clin Neurophysiol 1998;109:154-60.

Pfurtscheller G, Krausz G, Neuper C. Mechanical stimulation of the fingertip can induce crusts of beta oscillations in sensorimotor areas. J Clin Neurophysiol 2001;18:559-64.

Rothwell J. Introduction: plans, strategies and actions. Control of human voluntary movement. London: Chapman \& Hall; 1994. p. 1-5.

Salenius S, Schnitzler A, Salmelin R, Jousmaki V, Hari R. Modulation of human cortical rolandic rhythms during natural sensorimotor tasks. Neuroimage 1997;5:221-8.

Shibasaki H, Sadato N, Lyshkow H, Yonekura Y, Honda M, Nagamine T, Suwazono S, Magata Y, Ikeda A, Miyazaki M. Both primary motor cortex and supplementary motor area play an important role in complex finger movement. Brain 1993;116:1387-98. 
Singer W. Synchronization of cortical activity and its putative role in information processing and learning. Annu Rev Physiol 1993;55: 349-74.

Tallon-Baudry C, Bertrand O, Delpuech C, Pernier J. Stimulus specificity of phase-locked and non-phase-locked $40 \mathrm{~Hz}$ visual responses in human. J Neurosci 1996;16:4240-9.

Terzuolo CA, Viviani P. Determinants and characteristics of motor patterns used for typing. Neuroscience 1980;5:1085-103.
Toma K, Nagamine T, Yazawa S, Terada K, Ikeda A, Honda M, Oga T, Shibasaki H. Desynchronization and synchronization of central $20-\mathrm{Hz}$ rhythms associated with voluntary muscle relaxation: a magnetoencephalographic study. Exp Brain Res 2000; 134:417-25.

Tuller B, Kelso JAS, Harris KS. Interarticulator phasing as an index of temporal regularity in speech. J Exp Psychol: Hum Percept Perform 1982;8:460-72. 